\title{
Numerical Investigation of Mechanical Induced Stress During Precision End Milling Hardened Tool Steel
}

\author{
Andreas Reimer ${ }^{1, a^{*}}$, Stephen Fitzpatrick ${ }^{2, b}$, Xichun Luo ${ }^{1, c^{*}}$ and Jie Zhao ${ }^{1, d}$ \\ ${ }^{1}$ Centre for Precision Manufacturing, Department of Design, Manufacture and Engineering \\ Management, University of Strathclyde, UK \\ ${ }^{2}$ Advanced Forming Research Centre, University of Strathclyde, UK \\ aandreas.reimer@strath.ac.uk, bs.fitzpatrick@strath.ac.uk, cxichun.luo@strath.ac.uk, \\ j.zhao@strath.ac.uk
}

Keywords: FEM, Milling, tool steel, HSM, Residual Stress, Surface Integrity.

\begin{abstract}
Hardened tool steels are widely used materials for forming dies, due to their increased strength and hardness. However, their machinability is very poor, due to the high hardness of the material, which leads to high cutting forces and premature failure of the cutting tools. This is also associated with machining induced tensile stresses within the work piece. No full factorial design has been performed when end milling tool steel, due to the high associated costs. Instead of physical experiments, numerical models are commonly used to save cost and time. However, most of the recent research focus was only on 2D FE-Models. 2D model can be used for simulation of some simplified process, however, the results are not sufficient for accurate prediction. Therefore, a 3D FE-model of a precision end milling process with a two-flute ball nose cutter were established in this paper, in order to build a multi cutting edge model. In the FE-Model, a subroutine was implemented to model work piece hardening during the cutting process. The subroutine realised an accurate prediction of the residual stress and cutting forces. In addition, a material removal criterion was developed and implemented. The influence of cutting parameters on cutting force for end milling H13 tool steel was studied, through full factorial numerical simulations, to evaluate the effectiveness of this FEA model. Subsequently, after validation of the FEM model through machining trials, empirical models were developed for predicting cutting forces and residual stress. The cutting parameters evaluated were cutting speed, feed rate and depth of cut. In summary, it was found that the simulation and the experiments had a good agreement on the value and trend of the residual stress. The FEM model can be effectively used to predict residual stress in the machined surface.
\end{abstract}

\section{Introduction}

High speed machining process for hard materials has economic and technology benefits such as reduced process time and higher accuracy, compared to conventional machining. Often tool steel such as AISI H13 are used to produce forming tools for a broad range of industries, such as aerospace, automotive, end-consumer goods etc. This hardened steel can be cut by a high speed cutting machine with a high rigidity. Adiabatic heating occurs during high speed milling, which causes non-favourable tensile stresses on the work piece surface and subsurface which will influence the fatigue resistance of the work piece [1].

The aim of this research is to develop a framework and prediction model for the residual stresses in the work piece. The work included in this paper represents the primary stage of this research, to validate the 3D-FEM (Finite Element Model) in its residual stress state, and develop a t prediction model for residual stress and cutting forces.

The investigation of cutting strategies and surface integrity will support the optimisation of the cutting process, and enhance the fatigue resistance of the machined work pieces. There has been recently published work on milling hard / difficult to cut materials, such as H13 [2-5]. However, the published work did not exceed more than 21 physically or simulated experiments. Instead of limited 3D finite element (FE) models, 2D-models are dominating the published works, due to one dimension simplification, reduced calculation time, complexity and costs. 
This work is a 3D-FE-Model and framework, including a subroutine, for a more comprehensive material model. The advantage is that, the $3 \mathrm{D}$ model is able to represent a multi-cutting edge engagement during the cutting process, which has a major impact on cutting force, stress and temperature.

Three major influencing factors, i.e. feed rate, cutting speed and depth of cut are taken into account in this paper. The design of experiment (DOE) is conducted with three, four and five levels respectively, which lead to sixty FE-simulations in a full factorial experiment. In order to compare and validate the process simulation, nine strategies are physically machined. Cutting force is recorded by a Kistler dynamometer during the machining process. The surface residual stress, surface roughness and hardness are measured as well on each machined surface. Based on the validated simulation, prediction models for both residual stress and cutting forces are established.

\section{FEM-Model}

The milling simulation setup in ABAQUS 6-14 is shown in Figure 1. The cutting tool was set as rigid to reduce calculation time. The work piece was meshed in an explicit environment by using C3D8RT elements, which are 8-node thermally coupled brick with trilinear displacement, temperature, reduced integration and hourglass control elements.

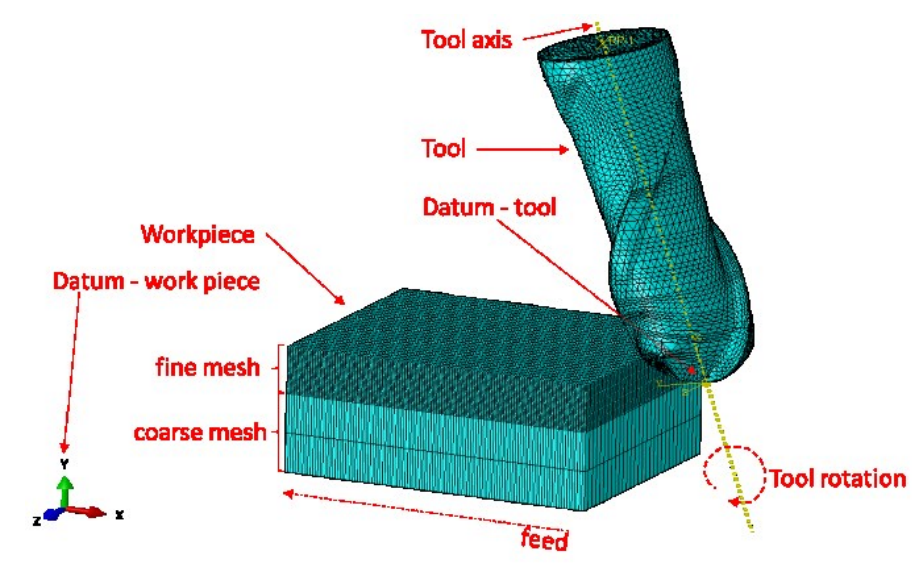

Figure 1: FEM milling setup

The work piece was sectioned into a fine mesh and coarse mesh area. Fine mesh had around 13000 elements and was used in the cutting region. AISI H13 material was chosen with an average hardness of 46 HRC.

In order to derive the stress and strain in the simulation, the equation of Johnson-Cook was initially implemented in ABAQUS. This equation is usually used for dynamic problems with high strain rates and temperature effect. The Johnson-Cook equation can realise a good macroscopic material description. However, in order to represent a more accurate material behaviour at microscopic level, the equation is extended by consideration of the hardness [4] as shown in equation (1).

$$
\bar{\sigma}(\bar{\varepsilon}, \dot{\bar{\varepsilon}}, T)=\left(A+B(\bar{\varepsilon})^{n}+D \ln \left(\varepsilon_{0}+\varepsilon\right)+E\right) \cdot\left(1+C \ln \frac{\dot{\bar{\varepsilon}}}{\dot{\dot{\varepsilon_{0}}}}\right) \cdot\left[1-\left(\frac{T-T_{0}}{T_{m}-T_{0}}\right)^{m}\right]
$$

The flow stress $\bar{\sigma}$ can be combined by the Von-Mises yield criterion and describes an isotropic hardening as follows in equation (1), where $\bar{\varepsilon}$ is the proportional strain, $\dot{\bar{\varepsilon}}$ is the proportional strain rate and $\mathrm{T}$ is the temperature. $\mathrm{A}, \mathrm{B}, \mathrm{C}, \mathrm{m}, \mathrm{n}, \mathrm{T}_{0}, \mathrm{~T}_{\mathrm{m}}$ are material parameters in the Johnson-Cook equation, $\varepsilon_{0}$ is the reference strain and $\varepsilon$ the current strain, these parameter can be found in [4-7]. The parameter $\mathrm{D}$ and $\mathrm{E}$ can be determined through a $2^{\text {nd }}$ and $3^{\text {rd }}$ grade of polynomial regression. Table 1 stated parameters used in FEM to determine the Johnson-Cook flow stress. 
Table 1: Johnson-Cook Parameter for H13

\begin{tabular}{ccccccccc}
\hline Material & Hardness [HRC] & A [MPa] & B [MPa] & C [-] & $\mathbf{n}[-]$ & $\mathbf{m ~}_{[-]}$ & $\mathbf{T}_{\mathbf{m}}\left[{ }^{\circ} \mathbf{K}\right]$ & Source \\
\hline $\mathrm{H} 13$ & 46 & 674.8 & 239.2 & 0.027 & 0.28 & 1.3 & 1,760 & {$[6]$} \\
\hline
\end{tabular}

This material hardening model written was in an ABAQUS subroutine VUHARD, which is similar to Umbrello's model [5], but an additional material removal criterion was added, which is based on the ultimate tensile strength (UTS). The UTS will change with temperature. It is calculated by using a $3^{\text {rd }}$ grade polynomial regression equation of tensile stress under different temperatures while starting from the room temperature in the FEM. If the flow stress exceeds the UTS, the elements are forced to be deleted, unless a separation criterion within ABAQUS has been applied before. The flow chart to demonstrate the principle of element removal criterion can be found in Figure 2.

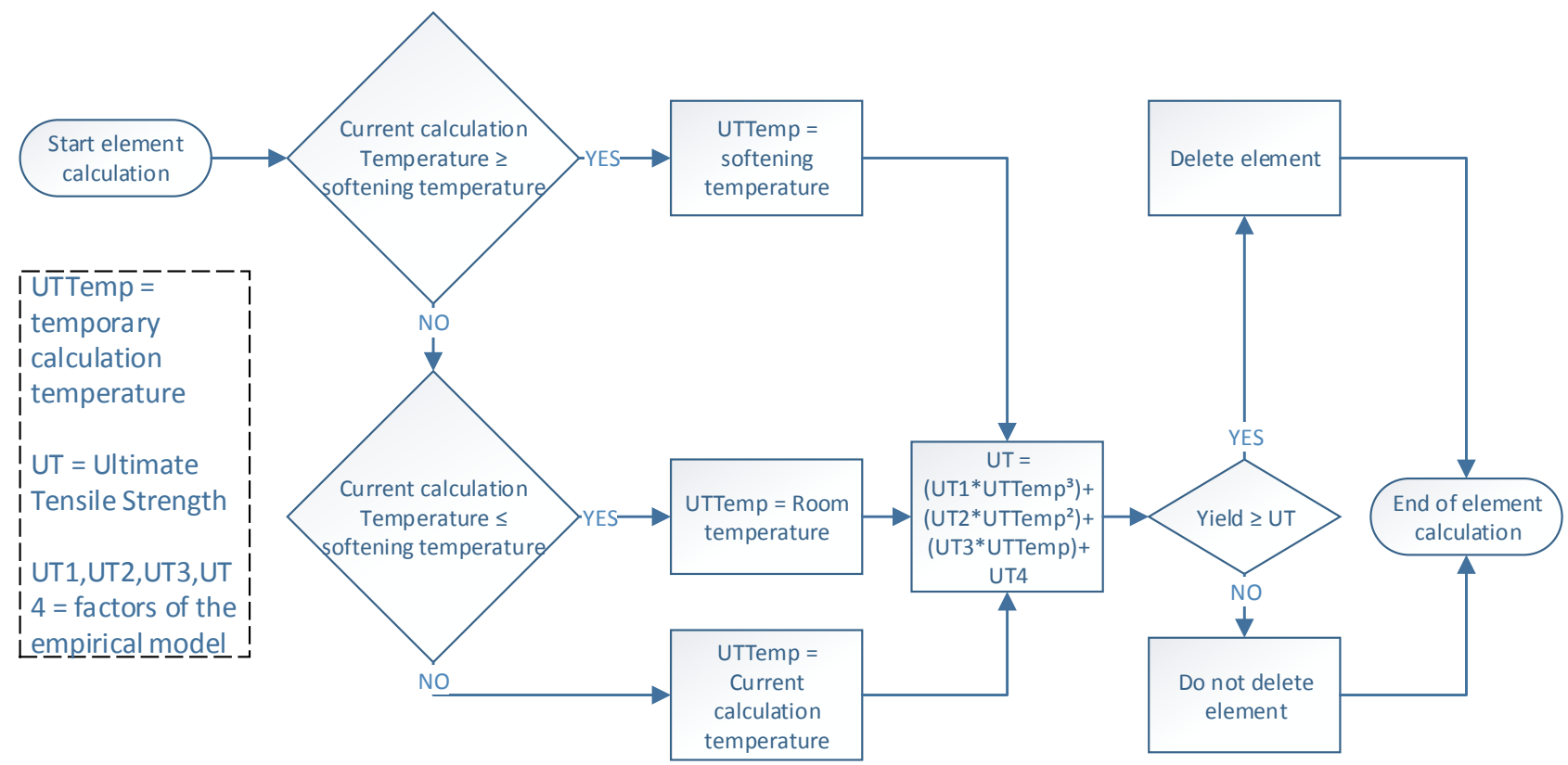

Figure 2: UTS deletion criteria principle

The full investigation plan was setup as follows. Cutting speed (Vel) with 4 levels, depth of cut (Doc) with 5 levels and feed rate (FR) with 3 levels, as (Table 2). In the following of the paper, the factors and their levels are stated in the sequence of cutting speed, depth of cut and feed rate (VelDoc-FR).

Table 2: Factors and applied levels

\begin{tabular}{l|ccccc}
\hline Factor / Level & $\mathbf{1}$ & $\mathbf{2}$ & $\mathbf{3}$ & $\mathbf{4}$ & $\mathbf{5}$ \\
\hline Doc $[\mathbf{m m}]$ & 0.2 & 0.3 & 0.4 & 0.5 & 0.6 \\
\hline Vel $[\mathbf{m} / \mathbf{m i n}]$ & 150 & 200 & 250 & 300 & \\
\hline FR $[\mathbf{m m} / \mathbf{t o o t h}]$ & 0.05 & 0.1 & 0.15 & & \\
\hline
\end{tabular}

\section{Experimental Work}

In order to validate the simulation results, the Taguchi Design of Experiment (DoE) method was used with nine physical experiments with same setup condition as the FEM. The setup levels of each experiment can be seen in Table 3 . 
The experimental setup is shown in Figure 3. A DMG HSC 75 machine was used for the machining, and a Kistler 9257BA dynamometer was used to record the cutting forces during the experiment.

Table 3: Experiment plan showing the factors and the applied levels

\begin{tabular}{c|ccc}
\hline Run [Vel-Doc-FR] & Cutting speed & Depth of Cut & Feed Rate \\
\hline $\mathbf{1 - 1 - 1}$ & 1 & 1 & 1 \\
\hline $\mathbf{1 - 2}-\mathbf{2}$ & 1 & 2 & 2 \\
\hline $\mathbf{1 - 5}-3$ & 1 & 5 & 3 \\
\hline $\mathbf{2 - 1 - 2}$ & 2 & 1 & 2 \\
\hline $\mathbf{2 - 2 - 3}$ & 2 & 2 & 3 \\
\hline $\mathbf{2 - 5 - 1}$ & 2 & 5 & 1 \\
\hline $\mathbf{4 - 1 - 3}$ & 4 & 1 & 3 \\
\hline $\mathbf{4 - 2 - 1}$ & 4 & 2 & 1 \\
\hline $\mathbf{4 - 5 - 2}$ & 4 & 5 & 2 \\
\hline
\end{tabular}

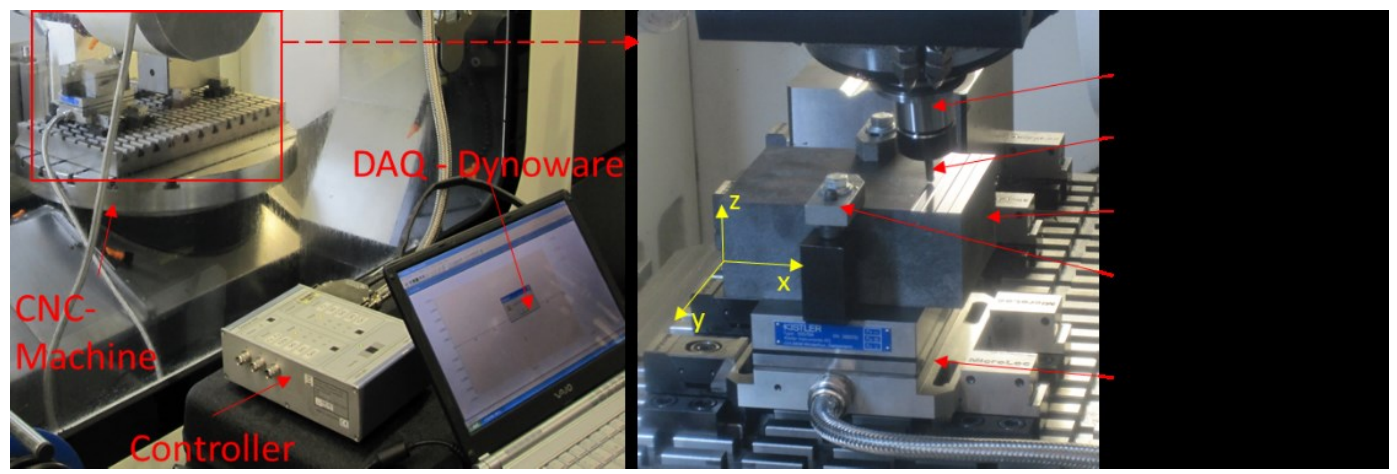

Figure 3: Experimental setup of the milling process

The cutting tool used was a $6 \mathrm{~mm}$ diameter tungsten carbide two-flute ball nose cutter, with a lead angle of $15^{\circ}$. Firstly, the work piece was cut one by one according to the DoE (Table 3 ). Then the residual stress was analysed on the cut work piece with the XRD (X-Ray Diffraction)-Method.

For the XRD Analysis, a Proto LXRD with a Chromium (Cr) tube in a Bragg-angle of $156.4^{\circ}$ was setup. Each experimental trial was measured 6 times, and an average was created in both longitudinal and perpendicular to the cutting direction. After the XRD-Analysis, the machined surface roughness (Ra) of work pieces was measured by an optical instrument -Alicona Infinite Focus microscope. Subsequently, the hardness was measured with a Streuers DuraScan 70 G5 hardness tester, with 4 indentations per machined strategy.

\section{Results and Discussion}

Simulation. The simulation environment was setup in a python script to maintain the same setup standard throughout all simulations. The behaviour of cutting forces influenced by the different cutting strategies were analysed and published in [8]. It was found that, the additional criterion of the material removal in the FEM, were leading to a good correlation to predict cutting forces. Based on the good correlation of the FEM and the cutting force, the model was also applied to predict residual stress.

The statistical significance factors (p-value) are $3.7 \cdot 10^{-10}$ for depth of cut, 0.0004 for feed rate and 0.459 for cutting speed. Since smaller values signify increased significance, it can be seen that depth of cut was the most influencing factor in residual stress generation, followed by feed rate and cutting speed. 
Experiment. The surface integrity was analysed, including residual stress, hardness and surface roughness. LXRD measured stress vectors and also the shear stress in each point. The measurement of the two stress vectors and the shear stress allows the calculation of the Von-Mises plane stress. The Von-Mises plane stress results can be seen in Figure 4. The experiment showed that the depth of cut was the most influencing factor, followed by feed rate and cutting speed.

Table 4 shows the measured results. The surface roughness ( $\mathrm{Ra}$ ) was measured in $\mu \mathrm{m}$ and hardness was measured in Vickers of HV10 (which was then converted to HRC).

Table 4: Machined surface roughness and hardness of the work pieces

\begin{tabular}{c|cc}
\hline Run [Vel-Doc-FR] & Surface Roughness (Ra) [ $\boldsymbol{\mu m}]$ & Hardness [HRC] \\
\hline $1-1-1$ & 0.393 & 36.4 \\
\hline $1-2-2$ & 0.571 & 35.6 \\
\hline $1-5-3$ & 0.517 & 34.2 \\
\hline $2-1-2$ & 0.581 & 40.5 \\
\hline $2-2-3$ & 0.627 & 40.8 \\
\hline $2-5-1$ & 0.371 & 40.9 \\
\hline $4-1-3$ & 0.592 & 41.0 \\
\hline $4-2-1$ & 0.456 & 40.1 \\
\hline $4-5-2$ & 0.491 & 43.3 \\
\hline
\end{tabular}

P-values of surface roughness and hardness can be found in Table 5, in which with the smallest numbers showing high level of significance. For machined surface roughness, it was found that the feed rate had the most significant impact, followed by the depth of cut and cutting speed on machine surface roughness. However, analysis of the hardness showed that the cutting speed had the most significant impact, followed by the feed rate and depth of cut.

It was observed that, the surface roughness had an opposite behaviour to the cutting force. Decreasing surface roughness causes increasing cutting forces. It was also found that, a general trend between the hardness and surface roughness can be correlated. That is to say increasing surface roughness will accompanied by increases hardness.

Table 5: P-values for surface roughness and hardness

\begin{tabular}{c|cc}
\hline Factor & Surface Roughness & Hardness \\
\hline Cutting speed & 0.687 & 0.011 \\
\hline Depth of Cut & 0.218 & 0.919 \\
\hline Feed Rate & 0.012 & 0.770 \\
\hline
\end{tabular}

Comparison. Figure 4 shows the values of residual stress in terms of Von-Mises plane stress obtained from both experiment and FEM. The measured residual stress from experiment setup 4-1-3 and 4-2-1 are higher than those calculated FEM. shown as two peaks. This abnormality in the physical experiment can be explained by the irregularity within the material, especially as both cutting trials were next to each other. However, most of the experimental results had a good correlation and similar trend with the simulation results. An average difference of $8.72 \%$ can be seen, which suggests generally good correlation between FEM and experiment. 


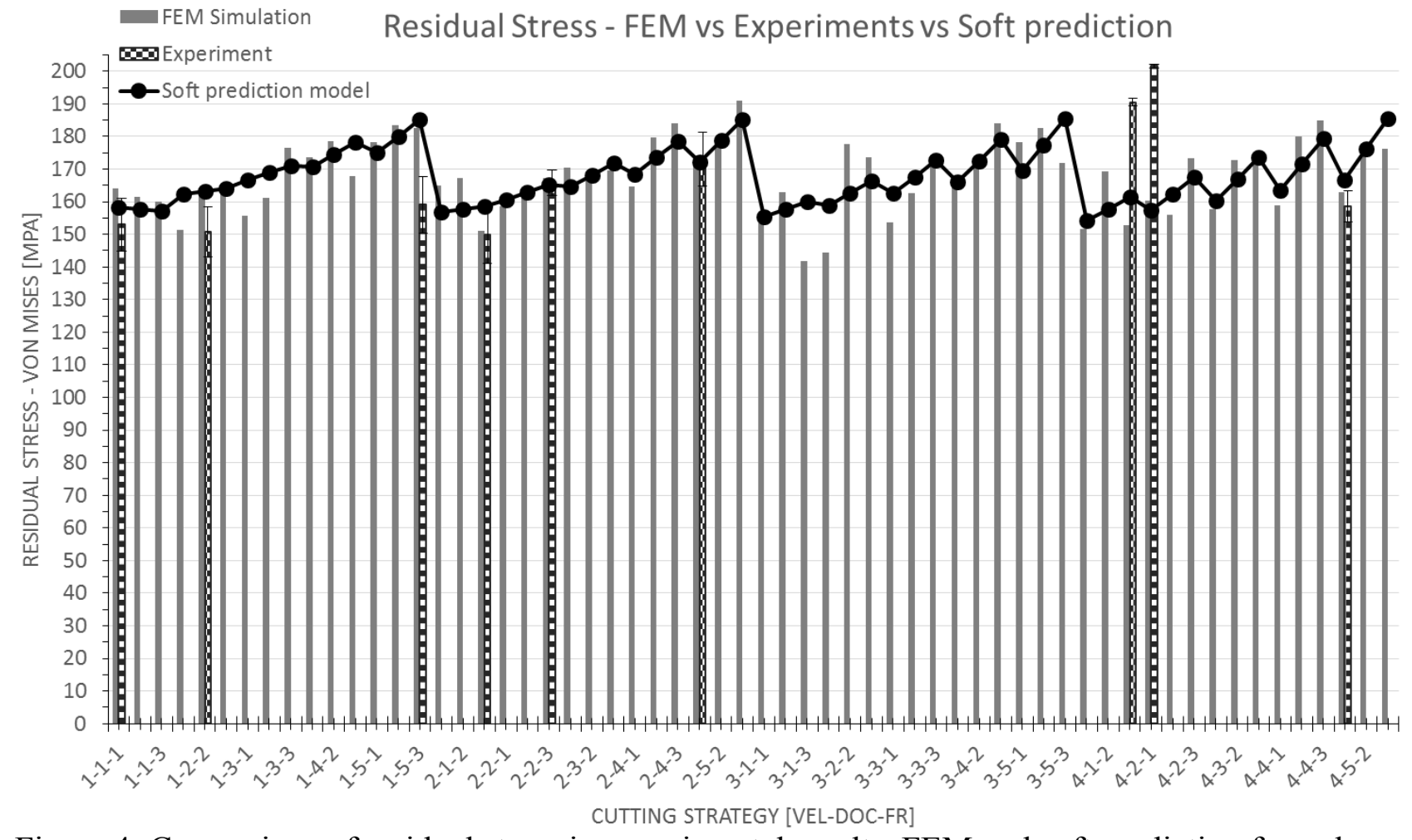

Figure 4: Comparison of residual stress in experimental results, FEM and soft prediction formula

Prediction model. Based on the FEM results, a comprehensive regression analysis was carried out to predict the residual stress and cutting forces, which can be described as:

$$
\begin{aligned}
& \sigma_{p}=159.382-0.041 \cdot \mathrm{Vel}+38.298 \cdot \mathrm{Doc}-152.927 \cdot \mathrm{FR}-0.07 \cdot \mathrm{Vel} \cdot \mathrm{Doc}+ \\
& 0.567 \cdot \mathrm{Vel} \cdot \mathrm{FR}+283.69 \cdot \mathrm{Doc} \cdot \mathrm{FR}
\end{aligned}
$$

and

$$
\begin{aligned}
& c f=12.102-0.054 \cdot \mathrm{Vel}+20.691 \cdot \mathrm{Doc}-156.346 \cdot \mathrm{FR}-0.007 \cdot \mathrm{Vel} \cdot \mathrm{Doc}+ \\
& 0.329 \cdot \mathrm{Vel} \cdot \mathrm{FR}+1696.365 \cdot \mathrm{Doc} \cdot \mathrm{FR}
\end{aligned}
$$

The predicted residual stresses shown in Figure 5 have average differences of $3.28 \%$ with the FEModel, and $8.78 \%$ with the experiments. It indicates that equation (2) can accurately predict the residual stress.

Similarly, as shown in Figure 5, the predicted cutting forces, based on equation (3), have good matches to those calculated by FEM, with an average difference of $11.95 \%$. 


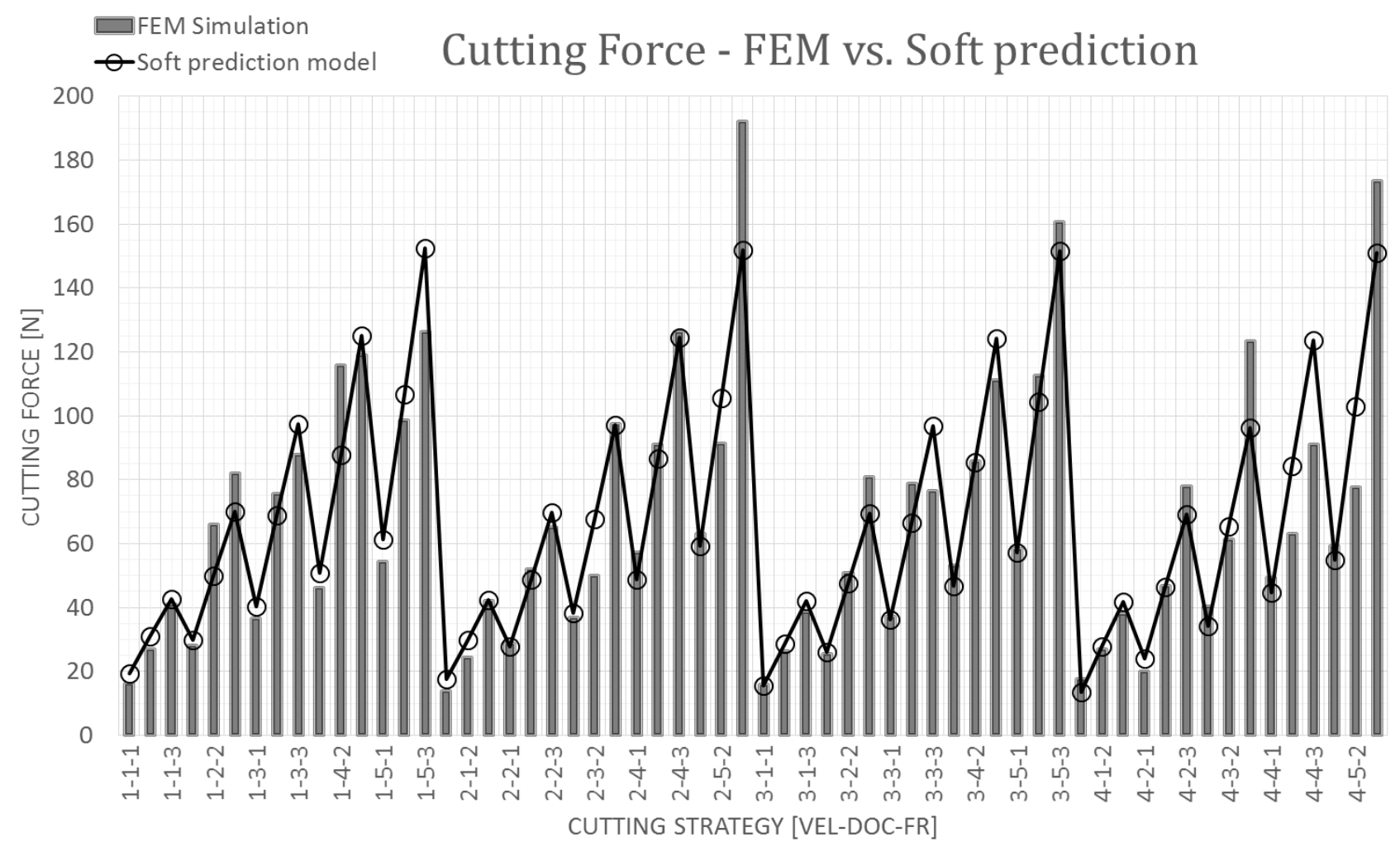

Figure 5: Comparison of cutting force in FEM and soft prediction formula

\section{Conclusion}

This work investigated a new approach to FE-Modelling of machining hard tool materials, by using an additional material removal criterion. Comparing the FE and experiment results it can be concluded that, the FEM setup is an effective model to predict residual stress, which is known to have an influence on surface integrity. Additionally it was found, that the depth of cut plays the most significant role in the residual stress, whereby it is the least influencing factor for the hardness.

The validated FE-Model also supported the development of prediction models for residual stress of cutting force where a good correlation difference of around 3\% for residual stress and less than $12 \%$ for the cutting force were achieved.

The benefit of the prediction models is to predict accurately residual stress (Von-Mises) and cutting forces in the given range of parameters, without carrying out time-consuming and costly experiments and simulations.

This work will contribute to academic as well as industries. Examples of benefiters are given below:

- Advanced development of a FE-Model for prediction models for residual stress and cutting forces for an end-milling process of a tool steel material.

- Validated 3D FE model for milling operation to calculate in cutting forces and residual stress.

- Identified the influencing factors of a 5-axis high speed ball nose end-milling process.

- Development of a validated empirical prediction model to accurately predict cutting force and residual stress.

It is worth noting that, the work mentioned in this paper is still the initial stage of the entire research. In the future, more physical experiments will be realised to fully validate the simulation. The predication models will also be further developed. The analysis and prediction of residual stress after machining and other related result will be published in the coming papers.

\section{Acknowledgement}

The authors would like to acknowledge the financial support from the Advanced Forming Research Centre (AFRC), UK and the EPSRC (EP/K018345/1) for this study. 


\section{References}

[1] Davim J P, Machining: fundamentals and recent advances. 2008: Springer Science \& Business Media.

[2] Axinte D and Dewes R, High-speed milling of AISI H13 hot-work tool steel using polycrystalline cubic boron nitride ball-nose mills: From experimental investigations and empirical modelling to functional testing of the machined surfaces. Proceedings of the Institution of Mechanical Engineers, Part B: Journal of Engineering Manufacture, 2010. 224(1): p. 15-24.

[3] Sulaiman S, Roshan A, and Borazjani S. Finite Element Modeling and Simulation of Machining of Titanium Alloy and H13 Tool Steel Using PCBN Tool. in Applied Mechanics and Materials. 2013. Trans Tech Publ.

[4] Yan H, Hua J, and Shivpuri R, Flow stress of AISI H13 die steel in hard machining. Materials \& Design, 2007. 28(1): p. 272-277.

[5] Umbrello D, et al., Hardness-based flow stress for numerical simulation of hard machining AISI H13 tool steel. Journal of Materials Processing Technology, 2008. 199(1-3): p. 64-73.

[6] Shatla M, Kerk C, and Altan T, Process modeling in machining. Part I: determination of flow stress data. International Journal of Machine Tools and Manufacture, 2001. 41(10): p. 15111534.

[7] Shrot A and Bäker M, Determination of Johnson-Cook parameters from machining simulations. Computational Materials Science, 2012. 52(1): p. 298-304.

[8] Reimer A, Fitzpatrick S, and Luo X, A full factorial numerical investigation and validation of precision end milling process for hardened tool steel, in Euspen's 17th Internation Conference \& Exhibition. 2017, Euspen: Hannover, Germany. 